\title{
Influence of timing of maternal antibiotic administration during caesarean section on infant microbial colonisation: a randomised controlled trial
}

\author{
Thomas Dierikx (D) , ${ }^{1,2}$ Daniel Berkhout, ${ }^{1,2}$ Anat Eck, $^{3}$ Sebastian Tims, ${ }^{3}$ \\ Johan van Limbergen (D) , ${ }^{2,4}$ Douwe Visser, ${ }^{5}$ Marjon de Boer, ${ }^{6}$ Nanne de Boer, ${ }^{7}$ \\ Daan Touw, ${ }^{8,9}$ Marc Benninga, ${ }^{2}$ Nine Schierbeek, ${ }_{1}$ Laura Visser, $^{6}$ Jan Knol, ${ }^{3,10}$ \\ Guus Roeselers, ${ }^{3}$ Johanna de Vries, ${ }^{6}$ Tim de Meij $^{1,2}$
}

Additional supplemental material is published online only. To view, please visit the journal online (http://dx.doi.org/ 10.1136/gutjnl-2021-324767).

For numbered affiliations see end of article.

\section{Correspondence to}

Thomas Dierikx, Department of Paediatric Gastroenterology, Amsterdam UMC Locatie VUmc, Amsterdam, The Netherlands: t.dierikx@amsterdamumc.nl

$\mathrm{JdV}$ and $\mathrm{TdM}$ are joint senior authors.

Received 26 March 2021 Accepted 2 November 2021

\section{Check for updates}

(c) Author(s) (or their employer(s)) 2021. Re-use permitted under CC BY-NC. No commercial re-use. See rights and permissions. Published by BMJ.

To cite: Dierikx T,

Berkhout D, Eck A, et al. Gut Epub ahead of print: [please include Day Month Year]. doi:10.1136/

gutjn-2021-324767

\section{ABSTRACT}

Objective Revised guidelines for caesarean section (CS) advise maternal antibiotic administration prior to skin incision instead of after umbilical cord clamping, unintentionally exposing the infant to antibiotics antenatally. We aimed to investigate if timing of intrapartum antibiotics contributes to the impairment of microbiota colonisation in CS born infants.

Design In this randomised controlled trial, women delivering via CS received antibiotics prior to skin incision $(n=20)$ or after umbilical cord clamping $(n=20)$. A third control group of vaginally delivering women $(n=23)$ was included. Faecal microbiota was determined from all infants at 1, 7 and 28 days after birth and at 3 years by 165 rRNA gene sequencing and whole-metagenome shotgun sequencing.

Results Compared with vaginally born infants, profound differences were found in microbial diversity and composition in both CS groups in the first month of life. A decreased abundance in species belonging to the genera Bacteroides and Bifidobacterium was found with a concurrent increase in members belonging to the phylum Proteobacteria. These differences could not be observed at 3 years of age. No statistically significant differences were observed in taxonomic and functional composition of the microbiome between both CS groups at any of the time points.

Conclusion We confirmed that microbiome colonisation is strongly affected by CS delivery. Our findings suggest that maternal antibiotic administration prior to CS does not result in a second hit on the compromised microbiome. Future, larger studies should confirm that antenatal antibiotic exposure in CS born infants does not aggravate colonisation impairment and impact long-term health.

\section{INTRODUCTION}

Early life microbiome acquisition and development can be compromised by external perturbations such as delivery via caesarean section (CS), formula feeding and antibiotics. ${ }^{1}$ Acute effects of antibiotics on the microbiota range from self-limiting diarrhoea to increased risk for life-threatening conditions in premature neonates. ${ }^{2} 3$ The longterm consequences of such perturbations for the

\section{Significance of this study}

What is already known on this subject?

- Microbial colonisation is essential for the development of the innate immune system. Early life gut microbiome acquisition and development can be compromised by external perturbations such as delivery via caesarean section (CS) and antibiotics.

What are the new findings?

- The present study confirms that CS delivery strongly affects microbiota development in the first month of life. Here we demonstrate that antenatal exposure to maternal administrated prophylactic antibiotics prior to skin incision, according to the current revised guidelines, does not seem to further aggravate the compromised microbiome development in CS born infants. Concluding that CS delivery itself, and not antenatal antibiotic exposure, seems to negatively affect microbiota development, and consequently infant health.

How might it impact on clinical practice in the foreseeable future?

- Given the ongoing worldwide increase in CS rates, larger prospective studies are needed to assess the relationship between observed CS-related disturbed colonisation and health outcomes later in life. Future studies are needed to confirm that beneficial effects for the mother by the revised guidelines do not impact longterm health of the infants.

human-microbial symbiosis are more difficult to discern, but chronic conditions such as IBD, obesity, asthma, allergy and type 1 diabetes have been associated with childhood antibiotic use and an altered intestinal microbiota. ${ }^{4-6}$

Over the last few years, international obstetric guidelines have been revised in order to reduce the incidence of maternal and neonatal infections. ${ }^{78}$ Because implementation of these adjusted guidelines has resulted in an increased use of antibiotics antenatally, ${ }^{78}$ concerns on early-life exposure to 
broad-spectrum antibiotics and associated pervasive effects on the gut microbiome development and various disorders later in life are growing. ${ }^{9}$ Besides, early-life antibiotic exposure may increase the risk of multi-resistant bacterial infections in neonatal patients. ${ }^{10}$ Recent epidemiological and mechanistic data on the association between early antibiotic use, dysbiosis and disease support these concerns. ${ }^{11}$ One of the revised protocols leading to an increased exposure to antibiotics worldwide is the National Institute for Health and Care Excellence (NICE) (2011) guideline for CS. ${ }^{7}$ In this revised guideline, it is advised to administer maternal prophylactic antibiotics prior to skin incision, instead of after clamping of the umbilical cord. This policy has been shown to reduce the maternal risk of infectious morbidities, particularly of endometritis and wound infections. ${ }^{12}$ Consequently, however, all infants born by CS are currently exposed to broad-spectrum antibiotics via the umbilical cord, when adhering to this revised guideline. Although no increase in incidence of neonatal sepsis was observed, ${ }^{12}$ effects on the gut microbiota colonisation and long-term health consequences remain largely unknown. We hypothesised that exposure to antibiotics in children delivered by CS, related to the revised international guidelines, influences the microbial colonisation process and may impact health outcome. In this randomised controlled trial (RCT), we evaluated this effect by comparing the microbiome composition of CS born infants with and without intrauterine antibiotic exposure, according to the revised and previous protocol, respectively, up to 3 years.

\section{METHODS}

\section{Study design}

This RCT was conducted at the obstetrics and paediatrics department of the Amsterdam UMC, location VUmc, a tertiary referral centre. Participants were recruited between March 2015 and November 2017. The study protocol of this study $(\mathrm{NTR} 6000)^{13}$ was approved by the ethics committee VUmc (2014.468). Written informed consent for participation was obtained from all parents. If parents declined participation, mothers received intrapartum antibiotic prophylaxis (IAP) after clamping of the umbilical cord according to the local hospital guideline. The trial is registered with the Dutch Clinical Trial Registry (Trial registration number: NTR6000, https://wwwtrialregisternl/trial/5845). The study protocol was published online (doi: 10.1186/s13063-019-3552-8). ${ }^{13}$

\section{Patient and public involvement}

Patients and public were not involved in the research question and the study design. Parents of all participants were contacted to evaluate relevant outcome measures and the burden of participation to improve future trials.

\section{Study population}

Pregnant women visiting outpatient clinics of the department of obstetrics and gynaecology during the third trimester of an uncomplicated pregnancy and scheduled for a primary CS were eligible to participate. Uncomplicated pregnancy was defined as a normotensive singleton pregnancy, with a normal-weight fetus, delivering at a gestational age $\geq 37$ weeks. An overview of all maternal and neonatal exclusion criteria is listed in box 1. Included women were randomly allocated to be treated according to the current or the previous NICE guideline on timing of prophylactic antibiotic administration during CS. The women treated according to the current NICE guideline $^{7}$ received $1500 \mathrm{mg}$ cefuroxime $30 \mathrm{~min}$ prior to CS (group

\section{Box 1 Maternal and neonatal exclusion criteria}

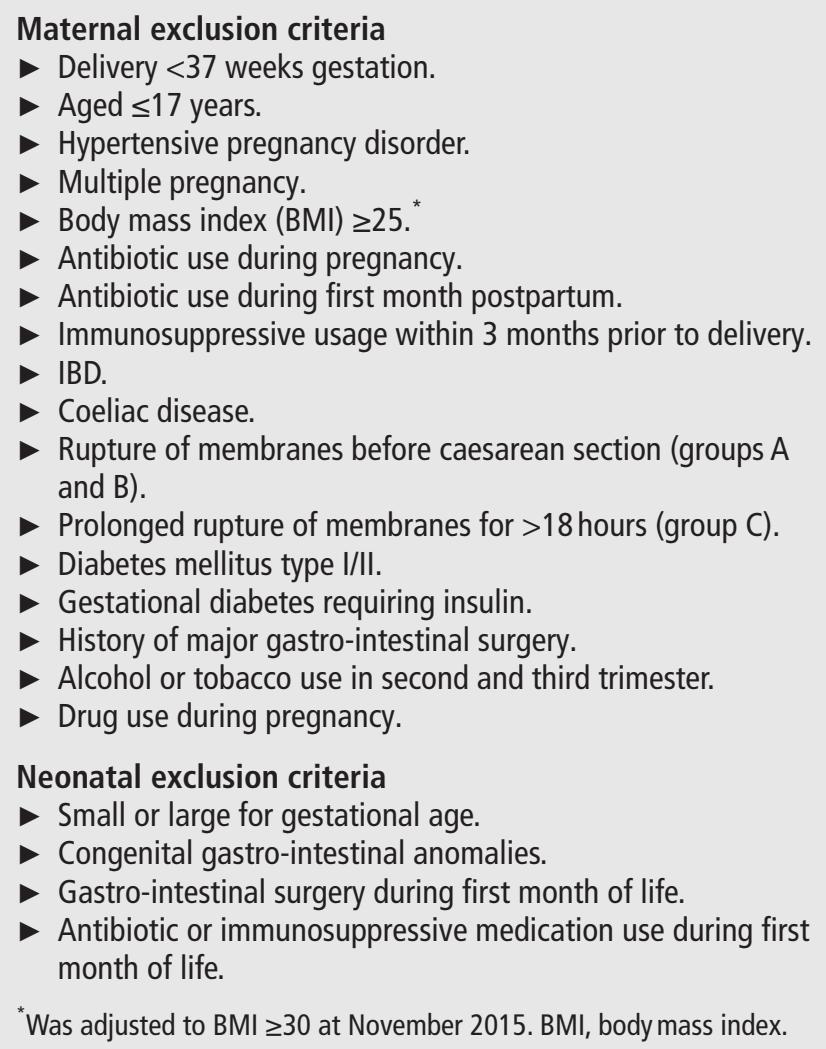

A). Those women allocated to be treated in accordance with previous NICE guideline, ${ }^{14}$ received $1500 \mathrm{mg}$ cefuroxime after clamping of the umbilical cord (group B). Randomisation was done by means of www.randomizer.org in permuted blocks of 10. A third control group of women visiting the outpatient clinic for vaginal delivery was included simultaneously during the study period, in order to compare CS with vaginally born infants (group C). The same eligibility criteria were retained for this group as for the two CS groups. Over time, the inclusion rate of the women delivering vaginally was adapted to the primary CS inclusions to facilitate inclusions in the same seasons.

\section{Blinding}

This study was not placebo controlled, since both groups received antibiotics; only the timing of antibiotics differed between groups A and B. The gynaecologist administering the antibiotics during CS was not blinded. However, the investigators analysing the samples and performing the statistics were blinded.

\section{Sample size calculation}

Since there is limited literature available on the influence of antibiotics during CS on infantile microbiota colonisation, ${ }^{15}$ a formal power analysis could not be performed for this study. We planned 20 inclusions per arm of investigation to enable detection of differences over time in line with the trial by Nogacka et al. ${ }^{16}$

\section{Sample and data collection}

Faecal sample collection

The first stool sample (meconium) was collected in a sterile container (Stuhlgefäß $10 \mathrm{~mL}$, Frickenhausen, Germany) by 
nurse or midwife, and immediately stored at $-20^{\circ} \mathrm{C}$. When discharged, parents were asked to collect faecal samples at home from their infants in provided containers at 7 and 28 days after birth. These samples were stored at home in a regular freezer and subsequently transported in cooled condition to the hospital on the day of the regular postpartum check-up 6 weeks after the delivery. At arrival in the hospital, the samples were collected by the investigator and stored at $-20^{\circ} \mathrm{C}$ until further handling. At the age of 3 years, parents collected a fourth faecal sample at home and stored them in a regular freezer. After collection, the faecal samples were transported in frozen condition to the hospital. At arrival in the hospital, the samples were stored at $-20^{\circ} \mathrm{C}$ until further handling.

\section{Umbilical cord blood collection}

To determine to what extent neonates were exposed to cefuroxime administered to the mother, umbilical cord blood was collected from infants of group A directly after clamping of the umbilical cord and delivery of the placenta. Blood samples were collected in an EDTA tube and directly transported to the laboratory. Samples were centrifuged and plasma was stored at $-80^{\circ} \mathrm{C}$ until the concentration of cefuroxime was determined.

\section{Data on health status}

Parents of all included infants were instructed to complete a questionnaire (online supplemental methods) at the age of 3 years. The questionnaire was slightly adjusted from a previously used questionnaire ${ }^{17}$ and included items on feeding practices, anthropometric measurements, medication and health-related problems like allergy, respiratory and gastro-intestinal symptoms.

\section{Sample handling}

DNA extraction

DNA from faecal samples of days 1,7 and 28 was extracted using the NucliSENS easyMag (bioMérieux, Marcy l'Etoile, France). NucliSENS lysis buffer $(1 \mathrm{~mL})$, containing guanidine thiocyanate, was added to a vial containing $150 \mu \mathrm{g}$ of faeces. The vial was shaken at $1400 \mathrm{rpm}$ (Thermomixer comfort, Eppendorf, Hamburg, Germany) for $5 \mathrm{~min}$ and consequently centrifuged for $4 \mathrm{~min}$ at $12000 \mathrm{~g}$. The vials were added to the easyMag container and DNA extraction was performed on the easyMag machine with the Specific A protocol as described by the manufacturer. Elution of DNA was performed using $110 \mu \mathrm{L}$ of buffer. Extracted DNA was stored at $4^{\circ} \mathrm{C}$ until further handling.

Due to merging of the laboratory and change in protocols, the follow-up samples collected at the age of 3 were analysed in a different laboratory compared with the neonatal samples, because of logistic reasons. The DNA was extracted using the QIAamp PowerFecal DNA Kit (Qiagen, Hilden, Germany). The DNA was extracted with minor adjustments of the manufacturer's protocol: for disruption of the samples, the TissueLyser II (Qiagen, Hilden, Germany) was used for $2 \mathrm{~min}$ at $30 \mathrm{~Hz}$. To increase the DNA concentration, $50 \mu \mathrm{L}$ of buffer solution was used instead of $100 \mu \mathrm{L}$ for the elution. Extracted DNA was normalised to $5 \mathrm{ng} / \mu \mathrm{L}$ and stored at $4{ }^{\circ} \mathrm{C}$ until use for PCR amplification.

\section{S rRNA gene sequencing}

All faecal samples were analysed using $16 \mathrm{~S}$ rRNA gene sequencing to characterise the taxonomic composition. V3-V4 hypervariable regions of the bacterial $16 \mathrm{~S}$ rRNA gene were amplified from the DNA extracted from faecal samples collected during the first month of life using universal primers S-D-Bact-0341-b-S-17 and S-D-Bact-0785-a-A-21. ${ }^{18}$ Sequencing was performed on an Illumina MiSeq instrument (Illumina, San Diego, California, USA) using the $2 \times 300 \mathrm{bp}$ paired-end sequencing protocol by LifeSequencing S.L. (Valencia, Spain). The read pairs were demultiplexed and trimmed ( $\mathrm{q}>20)$ before being merged using QIIME. ${ }^{19}$ Merged reads with $\mathrm{q}>25$ over a window of 15 bases, no ambiguous bases and a minimal length of 300 were retained. These were dereplicated and counted using mothur ${ }^{20}$ and reads with a low abundance (less than two reads over all samples) were discarded. Chimaeras were removed using VSEARCH ${ }^{21}$ using the RDP gold database ${ }^{22}$ as reference. Reads which contained PhiX or adapters as defined in Deblur (part of QIIME2) 2324 were eliminated. Taxonomic assignment was performed using the RDP classifier ${ }^{25}$ against the SILVA_ $119^{26}$ database, on basis of which the sequences,aggregated at genus and at phylum level, were further explored. Reads with eukaryotic assignments, as well as reads with a low relative abundance up to $0.0005 \%$ in all samples were excluded from further downstream analysis. Samples were rarefied, and $\alpha$-diversity was calculated using the phyloseq ${ }^{27}$ and vegan ${ }^{28}$ packages in R. ${ }^{29}$ On average 30921.4 sequences were generated per sample (ranging from 14216 to 91901 sequences; online supplemental table 1).

For the follow-up faecal samples collected at the age of 3, $16 \mathrm{~S}$ rRNA gene amplification and sequencing was done using the Earth Microbiome Project Protocol. ${ }^{30} 31$ The V4 region of the $16 \mathrm{~S}$ rRNA gene was amplified with a custom made 515F forward primer (Sigma-Aldrich, Saint Louis, Missouri, USA) and 806R reverse primer (Sigma-Aldrich) by using a one-step, singleindexed PCR approach. The library was paired-end sequenced $(2 \times 250 \mathrm{bp})$ on an Illumina MiSeq platform by the department of Cancer and Genomic Sciences at the University of Birmingham (Birmingham, UK). On average 59601.5 sequences were generated per sample (ranging from 22986 to 95091 sequences; online supplemental table 1).

\section{Whole metagenome shotgun sequencing}

Extracted DNA from samples of days 7 and 28 was used for whole metagenome shotgun (WMS) sequencing to further distinguish possible differences in more detail at these time points. These time-points were chosen since the effect of the perinatal antibiotics was expected to be most pronounced with limited influence of confounding environmental factors in these samples. In contrast to meconium, at day 7, the amount of human DNA will be decreased with a concurrent increase in DNA of the limited pioneer bacterial species present in the early microbiome. ${ }^{32}$ At day 28, the diversity will be increased due to an increased abundance of Veillonella, Streptococcus, Bifidobacterium and Enterobacteriaceae. ${ }^{33}$ Consequently, associations between perinatal factors and taxonomic composition are likely to be more pronounced after 1 month compared with early samples from the first week of life. ${ }^{32}$ DNA from samples collected at the age of 3 was not sequenced with WMS, since the microbiome had reached a more stable form ${ }^{34}$ and differences due to perinatal influences were expected to have disappeared by then.

Approximately $1-5 \mathrm{ng}$ of extracted DNA was used as input for the Illumina Nextera XT DNA Library Prep kit and barcoded using Nextera XT Indices, as per the manufacturer's instructions (Illumina, San Diego, California, USA). Isolated DNA was 'tagmented' (enzymatically 'sheared' and tagged with adaptors), single cycle PCR amplified to add barcodes, purified and normalised using Illumina beads. Final libraries were quantified using the Invitrogen Quant-iT dsDNA (high sensitivity) assay (Thermo Fisher Scientific) using a microplate reader, equal 
amounts of each library were pooled and then sequenced at the Integrated Microbiome Resource (Dalhousie University, Halifax NS) using $2 \times 150 \mathrm{bp} \mathrm{PE}$ reads on an Illumina NextSeq 550 using the High Output v2.0 chemistry. On average 9274349.4 sequences were generated per sample (ranging from 1076734 to 19473464 sequences; online supplemental table 1). Sequence reads were subjected to the MG-RAST pipeline (V.4.0.3) with default settings. ${ }^{35} 36$ Sequence reads were taxonomically classified by a sBLAT similarity search against the M5rna database which integrates the SILVA, ${ }^{26}$ Greengenes $^{37}$ and RDP $^{38}$ databases. Functional classification of the predicted proteins was performed by a sBLAT similarity search against the M5nr database, ${ }^{39}$ which provides non-redundant integration of many databases: GenBank, SEED, IMG, UniProt, KEGG and eggNOGs.

Antimicrobial resistance genes within the WMS dataset were predicted with the deep-learning approach, DeepARG. ${ }^{40}$ Translated fasta sequence files (all possible open-reading frames) were used as input for DeepARG. All potential antimicrobial resistance genes were identified using the Comprehensive Antibiotic Resistance Database ${ }^{41}$ with DeepARG.

\section{Umbilical cord blood}

Cefuroxime plasma concentrations $(\mathrm{mg} / \mathrm{L})$ were determined using a validated high performance liquid chromatography ultraviolet detection analysis at the department of Clinical Pharmacy and Pharmacology, University Medical Centre Groningen, The Netherlands. Validation was carried out according to European Medicines Agency guidelines. The lower limit of quantitation was $0.4 \mathrm{mg} / \mathrm{L}$ and upper limit of quantitation was $100 \mathrm{mg} / \mathrm{L}$. Variation coefficient was less than $4 \%$ over the entire working range.

\section{Statistical analysis \\ Demographic data}

Demographic data were given descriptively. For health outcome variables at the age of 3, comparisons of continuous variables between the three study groups were done using a one-way analysis of variance for normal distributed variables and KruskalWallis test for non-normal distributed variables. The $\chi^{2}$ test was used to compare dichotomous outcome variables. Differences were considered significant if the two-sided p value was $<0.05$.

\section{Statistical analysis 16S rRNA gene sequencing data}

At each time-point, differential abundance analysis of the detected taxa was performed with the Analysis of Composition of Microbiomes with Bias Correction (ANCOM-BC) (V.1.2.0) ${ }^{42}$ in R (V.4.1.0) with phyloseq (V.1.36). ANCOM-BC uses a linear regression framework in order to estimate the unknown compositional as well as sampling fractions from the sequence count data. Both the differences between all CS and vaginally born infants, as well as the differences between the two CS groups $\mathrm{A}$ and $\mathrm{B}$ were evaluated. The resulting large sets of $\mathrm{p}$ values were corrected for multiple testing by assessing the positive false discovery rate $e^{43}$ hence all reported $\mathrm{p}$ values are adjusted $\mathrm{p}$ values. The R-package ggplot2 (3.3.5) was used for visualisation.

Within-sample diversity was calculated using the Shannon diversity index on the genus level data for each group at each time point. Between-sample diversity was calculated based on Bray-Curtis distances on the genus level data, and the dissimilarity matrix was then used for the calculation of principal coordinate analysis (PCoA). The PCoA procedure was performed using Canoco 5 software for multivariate data exploration. ${ }^{44}$
Statistical analysis whole metagenome shotgun sequencing data Differential abundance analysis on the datasets resulting from the WMS sequencing was performed as described above with ANCOM-BC as well. Furthermore, at each time point the same approach was followed for the functionally annotated datasets.

All potential antimicrobial resistance gene (identitifed by DeepARG) counts were subjected to a Wilcoxon Rank Sum test to calculate the $\mathrm{p}$ value between the two CS groups A and B at day 7 and at day 28 .

\section{RESULTS}

\section{Patient population}

During the inclusion period 572 women delivered via a primary CS. After screening and randomisation, 20 women delivering via CS receiving antibiotic prophylaxis prior to skin incision (group A: antenatally antibiotic exposed infants) and 20 after clamping of the umbilical cord (group B: antenatally antibiotic unexposed infants) were included. A total of 23 women delivering vaginally were found eligible to participate in the vaginal control group (group C). The flow of patient selection and reasons for exclusion is given in figure 1. Demographic and clinical characteristics of included mothers and infants are shown in table 1 . None of the variables differed significantly between the study groups.

\section{Microbiome analysis}

16S rRNA based microbiome composition: vaginally versus CS born infants

Compared with vaginally born infants, both CS groups had a significant lower Shannon diversity at day $28 \quad(\mathrm{p}<0.001)$ (figure 2A). Figure 2B,C shows that the $16 \mathrm{~S}$ rRNA gene derived taxonomic composition of samples collected from vaginally born infants cluster to the exclusion of samples collected from CS born infants at days 7 and 28. In both CS groups, interindividual differences were apparent and seemed to prevail over potential antibiotic administration induced differences. In the beta diversity plots, principal coordinates from samples of the vaginal group also clustered together at day 28 , while samples of both CS groups did not (online supplemental figure 1). After 3 years, differences in Shannon diversity and the principal coordinate analysis had disappeared (online supplemental figure 1).

No differences in the microbiota were found on phylum level between vaginally and CS born infants on days 1 and 7 . Compared with vaginally born infants, the microbiota of CS born infants harboured a decreased abundance of Bacteroides $(\mathrm{p}<0.001)$ on day 28 with a concurrent increase in Firmicutes $(p=0.001)$ (online supplemental figure 2 and online supplemental dataset $1 \mathrm{~A}-\mathrm{C}$ ). At genus level, numerous differences were found including a decrease in Bacteroides with a concurrent increase in Enterococcus (online supplemental datasets 1D-F). At 3 years of age, no differences between vaginally and CS born infants were present at phylum nor genus level.

\section{Whole metagenome based microbiome composition: vaginally versus CS born infants}

At phylum level, a decrease in the abundance of Bacteroidetes and an increase of Lactobacillus was found in CS born infants at day 7. At day 28, also a decreased abundance in Bacteroidetes was present in CS born infants (online supplemental figure 3 and online supplemental dataset $2 \mathrm{~A}, \mathrm{~B})$.

At genus level, the microbiota of CS born infants harboured a decreased abundance of Bacteroides, Prevotella and Akkermansia compared with vaginally born infants at day 7. Furthermore, significant differences were found in the abundance of 13 other 


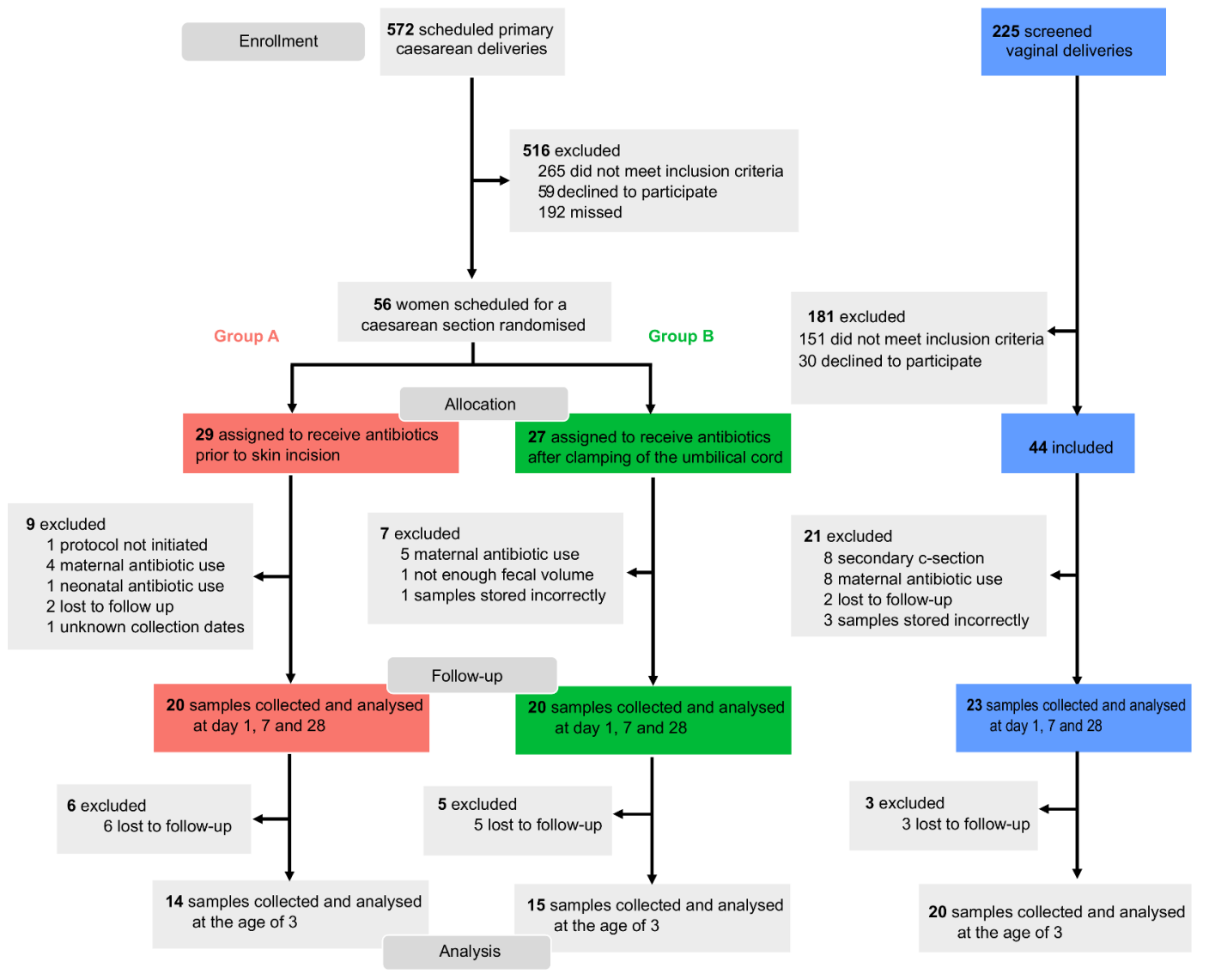

Figure 1 Consort diagram.

genera at day 7 (online supplemental dataset 2C). Also at day 28, the abundance of the genera Bacteroides, Prevotella and Akkermansia was decreased in CS born infants. The abundance of Klebsiella, Pseudomonas, Enterococcus, Clostridium and Enterobacter were significantly increased in CS born infants, along with changes in the abundance of 57 other genera at day 28 (online supplemental dataset 2D). On species level, there were significant differences in 118 and 188 species at days 7 and 28, respectively. These species did mainly belong to the previously mentioned genera and to members of the genus Bifidobacterium (online supplemental dataset 2E,F).

The abundance of numerous function genes did significantly differ between vaginally and CS born infants day 7 (133 genes) and day 27 (663 genes). An overview of these genes is depicted in online supplemental dataset $2 \mathrm{G}, \mathrm{H}$.

\section{S rRNA based microbiome composition: CS groups}

No differences in Shannon diversity indices were found at all four time points between the two CS groups (figure 2A). Beta diversity plots also showed no differences (online supplemental figure 1). A heatmap of samples collected at days 7 and 28 showed that the vaginal group clustered together, but did not demonstrate clear difference between the two CS groups (figure 2B,C). No differences were found in taxonomic composition between groups $\mathrm{A}$ and $\mathrm{B}$ at all four time points on phylum level (online supplemental figure 2) nor on genus and species level. An overview of the phyla and genera compared between the two CS groups based on the 16S sequence data along with adjusted $p$ values are demonstrated in online supplemental dataset 3A-F. Furthermore, no differences in (potential) antimicrobial resistance genes were found during the first month of life (online supplemental figure 4).

Whole metagenome based microbiome composition: CS groups At phylum level, no differences were found between the two CS groups at day 7 nor at day 28 (online supplemental figure 4 and online supplemental dataset 4A,B). Also at genus and species level, no significant differences were found between antenatally antibiotic exposed and unexposed CS born infants (online supplemental dataset 4C-F).

Analyses of subsystems (sets of functional roles that together implement a specific biological process or structural complex $)^{45}$ did not reveal any differences between both CS protocols. At days 7 (online supplemental dataset 4G) and 28 (online supplemental dataset $4 \mathrm{H}$ ) the abundance of none of the analysed functions did significantly differ between both CS groups.

Functionality was further investigated specifically for the (potential) antimicrobial resistance genes but these were not significantly different between the CS groups at day $7(p=0.88)$, nor at day $28(\mathrm{p}=0.20)$ (online supplemental figure 4$)$.

\section{Cefuroxime cord blood levels}

In 17 of 20 included infants of group A umbilical cord blood was analysed to determine cefuroxime levels. Two samples were excluded since two mothers received prophylactic clindamycin because of a suspected cefuroxime allergy and in one case the blood sample was collected incorrectly. The median cefuroxime level of the analysed samples was $13.7 \mathrm{mg} / \mathrm{L}$ (IQR $11.2-17.8 \mathrm{mg} / \mathrm{L}$ ), which is above the minimal inhibitory concentration (MIC) of most bacterial species. ${ }^{46}$ 
Table 1 Mother and infant baseline characteristics. Women delivering via caesarean section received antibiotics prior to skin incision (group A) or after clamping of the umbilical cord (group B). Comparison between both caesarean groups was done using the $\chi^{2}$ test or Fisher's exact test for dichotomous variables and Student's t-test or Mann-Whitney U for normally and non-normally distributed continuous data. Vaginally delivering women (group C) were included as a controls and were not exposed to antibiotics

\begin{tabular}{|c|c|c|c|c|}
\hline Characteristics & Group A $(n=20)$ & Group B $(n=20)$ & Group C ( $n=23)$ & $P$ value \\
\hline Maternal age at birth, median (IQR), years & $36.6(33.4-39.3)$ & $36.0(31.7-39.0)$ & $32.3(30.8-35.9)$ & 0.550 \\
\hline BMI, median (IQR), kg/m² & $22.8(19.8-24.3)$ & $23.8(21.2-25.0)$ & $21.9(20.8-23.3)$ & 0.594 \\
\hline Gravida, median (IQR) & $3(2-4)$ & $3(2-4)$ & $2(1-3)$ & 0.620 \\
\hline Para, median (IQR) & $1(1-1)$ & $1(0-2)$ & $1(0-1)$ & 0.779 \\
\hline \multicolumn{5}{|l|}{ Maternal diet at birth } \\
\hline Vegetarian & $1(5)$ & $1(5)$ & $3(13)$ & 0.970 \\
\hline Non-vegetarian & $18(90)$ & $19(95)$ & $20(87)$ & \\
\hline Missing & $1(5.0)$ & $0(0)$ & $0(0)$ & \\
\hline \multicolumn{5}{|l|}{ First or repeat caesarean section } \\
\hline First & $5(25)$ & $9(45)$ & NA & 0.185 \\
\hline Repeat & $15(75)$ & $11(55)$ & NA & \\
\hline Gestational age, median (IQR), weeks+days & $39+0(37+6-39+6)$ & $39+0(38+5-39+2)$ & $39+6(38+4-40+3)$ & 0.383 \\
\hline Birth weight, $g$ & $3518(380)$ & $3442(593)$ & $3385(484)$ & 0.634 \\
\hline \multicolumn{5}{|l|}{ Sex } \\
\hline Female & $12(60)$ & $7(35)$ & $14(61)$ & 0.113 \\
\hline Male & $8(40)$ & $13(65)$ & $9(39)$ & \\
\hline \multicolumn{5}{|l|}{$\mathrm{P}$ value birth weight } \\
\hline $\mathrm{p}<10$ & $0(0)$ & $3(15)$ & $0(0)$ & 0.341 \\
\hline p 10-50 & $8(40)$ & $6(30)$ & $11(48)$ & \\
\hline p 51-89 & $9(45)$ & $8(40)$ & $10(44)$ & \\
\hline$p>90$ & $3(15)$ & $3(15)$ & $2(9)$ & \\
\hline \multicolumn{5}{|l|}{ Apgar score, median (IQR) } \\
\hline $1 \mathrm{~min}$ & $9(9-9)$ & $9(9-9)$ & $9(8-9)$ & 0.947 \\
\hline $5 \mathrm{~min}$ & $10(10-10)$ & $10(10-10)$ & $10(9-10)$ & 0.862 \\
\hline Meconium stained amniotic fluid & $0(0)$ & $1(5)$ & $3(13)$ & 0.311 \\
\hline \multicolumn{5}{|l|}{ Feeding type } \\
\hline Breastfed & $10(50)$ & $10(50)$ & $15(65)$ & 0.403 \\
\hline Formula fed & $6(30)$ & $3(15)$ & $4(17)$ & \\
\hline Combination & $4(20)$ & $7(35)$ & $4(17)$ & \\
\hline
\end{tabular}

\section{Questionnaire 3 years after birth}

No differences were observed in the health status at the age of 3 years between the three groups (online supplemental table 2).

\section{DISCUSSION}

In this RCT, the effect of timing of maternal prophylactic antibiotic administration during CS on the microbiome and health state of infants up to 3 years of age was evaluated. Moreover, the findings were compared with a control group of vaginally born infants during the same time period. This study confirmed previous findings that CS delivery in general leads to a profound hit on the initial microbial colonisation. Our data suggest that maternal antibiotic administration prior to CS does not lead to a 'second hit' on the already compromised microbiome in CS born infants.

The rate of infants born by CS continues to increase worldwide. Currently, reported rates vary from around a quarter to more than half of all infants. ${ }^{47}$ In this study, besides a decreased diversity, the abundance of numerous phyla, genera and species was significantly affected by a CS delivery. The main aberrations found in the microbiota of CS born infants included a decreased abundance of members belonging to genera Bacteroides and Bifidobacterium and an increased abundance of Proteobacteria and enterococci during the first month of life. These findings are largely in line with previous studies demonstrating a decreased abundance of Bacteroidetes, ${ }^{48}$ decreased diversity ${ }^{49}$ and an increase in opportunistic pathogens, mainly including enterococci, in CS born infants. ${ }^{48} 49$ Knowledge about the development and impact of perinatal factors on species level is still limited. We confirmed findings by Saturio et $a l^{50}$ that CS born infants have a decreased abundance of B. bifidum and $B$. catenalutum, but we did not find evidence of increased abundance in other Bifidobacterium species such as B. adolescentis and B. animalis. The abundance of numerous species, mainly of the mentioned genera and phyla, was significantly affected by the route of delivery.

Alterations in microbiota colonisation have previously been associated with disturbed development of the immune system and longterm complications. ${ }^{4-6}$ Bifidobacteria and Bacteroides, for example, are considered to confer positive health benefits in general on the host. ${ }^{5152}$ Bifidobacteria produce acetate and lactate which act as a barrier against enteropathogenic infections. Delayed colonisation with bifidobacteria has been associated with a decreased number of memory B-cells later in infancy and with immune dysregulations, ${ }^{53}$ and an consequently with increased risk for multiple noncommunicable diseases later in life. ${ }^{51}$ Bacteroides also influence immune development, and depletion of this genus in infancy could negatively impact T-cell response. Proteobacteria comprise multiple known human pathogens. An increase in the abundance of Proteobacteria is seen in numerous clinical conditions. Furthermore, a microbiota depleted of Bacteroides with increased abundance of Proteobacteria during infancy has been associated with long-term complications including impaired neurocognitive development. ${ }^{54}$ 
A

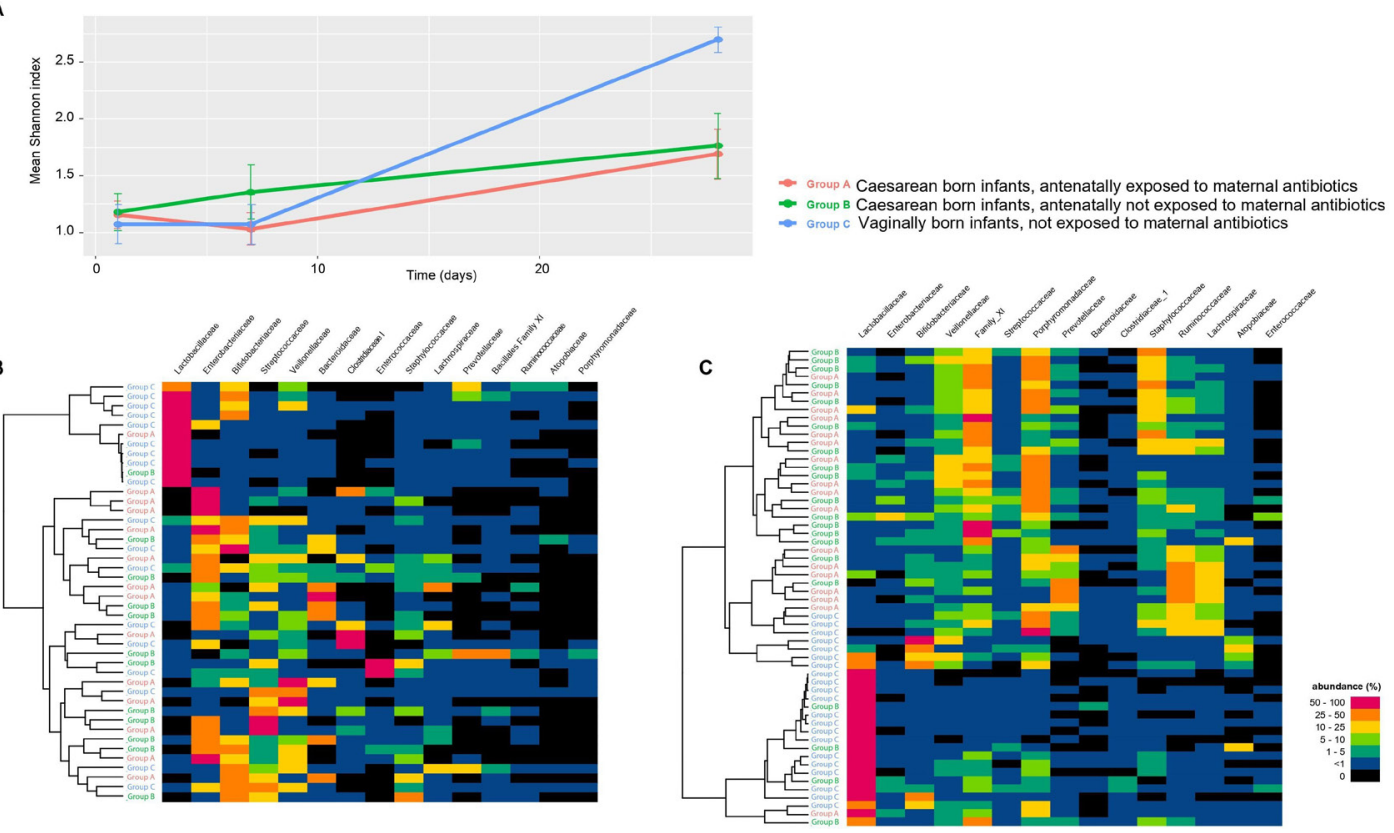

Figure 2 Mean Shannon diversity indices and taxonomic composition of the microbiota. (A) Mean Shannon diversity indices calculated from the taxonomic assignments (genus level) of the 16S rRNA gene sequence analyses of faecal samples collected at 1, 7 and 28 days postpartum from infants of mothers delivering via caesarean section who received prophylactic antibiotics either before skin incision (group A: antenatally antibiotic exposed infants) or after cord clamp (group B: antenatally antibiotic unexposed infants). Faecal samples were also collected from a third group of vaginally born infants (group C). Samples were analysed by 16S rRNA gene sequencing. At days 1 and 7 no significant difference was present between infants from all three groups. At day 7, mean Shannon diversity was 1.03 in group $A$ and 1.36 in group $B(p=0.23)$. At day 28 Shannon diversity index of vaginally born infants was significantly higher compared with both caesarean groups $(p<0.001)$. (B and $C)$ Left side dendrogram shows results of unsupervised cluster analysis of the taxonomic assignments (genus level) based on Bray-Curtis dissimilarity. Samples collected from vaginally born infant (group C) cluster to the exclusion of samples collected from caesarean section born infants (groups A and B). Right side; taxonomic composition of the microbiota demonstrated in a heat map of individual samples collected at day 7 (B) and day 28 (C) depicting the relative abundance (\%) of the 15 most abundant bacterial families.

Besides CS itself, it has been shown that postnatal antibiotics impact the abundance of keystone microbial taxa. ${ }^{55}$ Antibiotic exposure early in life decreases the diversity, the abundance of Bacteroides and Bifidobacterium species and increases the abundance of Enterobacteriaceae. ${ }^{56}$ Currently, it is unknown whether the effects of maternal IAP resemble effects of postnatal antibiotics on the microbiota in CS born infants, and further increases the risk for microbiota-related long-term health complications. In vaginally born infants, maternal IAP has been shown to decrease the diversity and abundance of Bacteroidetes and bifidobacteria and to increase the abundance of Proteobacteria, ${ }^{15}{ }^{16}$ which might increase the risk for negative long-term health outcomes. ${ }^{4-6}$ It might be counter-intuitive to assume negative effects of maternal IAP are only present in vaginally born infants and not in CS born infants. This is the first RCT evaluating effects on infant microbiota colonisation of exposure to maternal IAP during CS in a randomised design using metagenomics. Despite the high concentrations of cefuroxime measured in the umbilical cord and the fact that numerous species of the human gut microbiota are susceptible to cefuroxime, ${ }^{5758}$ we showed that intrauterine exposure to antibiotics does not result in a 'second hit' on the already compromised microbiome in CS born infants.

Only one previous RCT investigated the effect of timing of antibiotic administration during CS on the infant microbiota using $16 \mathrm{~S}$ rRNA gene sequencing. ${ }^{59}$ In that study, the effect on the infant microbiota was measured after 10 days and 9 months. In line with our findings, no differences were demonstrated in the taxonomic composition at 10 days postnatally, but a significantly decreased microbial species richness was found in intrauterine antibiotic exposed infants after 9 months. Besides 16S rRNA sequencing, we analysed samples using WMS. Both methods are substantially different and can yield quantitatively and qualitatively different results. ${ }^{60-64}$ The advantage of WMS is that it provides direct information about the presence or absence of specific microbial functions such as antibiotic resistance. ${ }^{60-64}$ Since it has been demonstrated that perinatal factors could influence the abundance of different species of the same genera in opposite directions (eg, an increase in $B$. bifidum and a simultaneous decrease in $B$. adolescentis following CS), the importance of analysis on species level, possible with WMS, is emphasised. ${ }^{50}$ Previous studies showed only a weak correlation between amplicon sequenced data and WMS sequencing data and this may explain why we observed differences in results between both methods. Discrepancies between the $16 \mathrm{~S}$ and WMS datasets might further be explained by PCR primer bias. ${ }^{65}$ Since both methods have their own advantages and are therefore considered as complementary, it is considered useful to analyse samples parallel with both techniques. ${ }^{60-64}$

Besides combined 16S rRNA amplicon sequencing and WMS sequencing, which allows taxonomic analysis up to species level and analysis of functional genes and antibiotic resistance genes, other strengths of this study include the randomised controlled study design for the CS group and inclusion of the vaginally born group. Application of strict inclusion and exclusion criteria limited the risk of bias and long follow-up period provides insight on long-term microbiota development. The cefuroxime cord blood concentrations in exposed neonates provided valuable information on the degree of antibiotic exposure. Despite the short exposure period of $30 \mathrm{~min}$, a median concentration of $13,7 \mathrm{mg} / \mathrm{L}$ could be found in the umbilical cord, which is above the MIC of most bacterial species. ${ }^{465758}$ Limitations 
of our trial include the relatively small sample size, hampering to draw firm conclusions regarding long-term health outcomes.

A reduction of maternal infectious morbidities was the reason for revising the recommendation regarding the timing of IAP in the NICE guidelines. ${ }^{712}$ Women receiving antibiotics prior to CS are affected in $3.9 \%$, predominantly by endometritis and wound infections, compared with $6.9 \%$ of women receiving antibiotics after cord clamp (risk ratio: 0.57 and number needed to treat: 33.3 ). ${ }^{12}$ Importantly, effects on neonatal gut microbial colonisation and longterm effects associated with antibiotic exposure have not been investigated before implementation of these adjusted guidelines. Notably, the majority of eligible parents preferred to be treated according to the previous NICE guidelines, considering the uncertain risk of antibiotic exposure more important than the proven protective effects on risk of maternal infection. Here, we have for the first time shown that adhering to the current NICE guidelines does not seem to significantly impact the infant faecal microbiome up to 3 years of age. Future studies should confirm the hypothesis that antenatal antibiotic exposure in CS indeed does not influence long-term health outcomes, like asthma, allergy and obesity. ${ }^{5}$ These studies could further reduce the uncertainty and doubts of parents and clinicians whether the beneficial protective effects for mother by the guideline adjustment do not lead to negative long-term consequences for the child and justify the guideline adjustment.

\section{CONCLUSIONS}

We confirmed that early-life microbiome development is strongly affected by mode of delivery. In this RCT, we observed that maternal antibiotic administration before onset of the CS according to the current guidelines, does not seem to further impact the compromised microbiota development in CS born infants. Disturbances in microbial colonisation have previously been associated with a disturbed priming of the immune system, even when these microbial disturbances are restored later in life. Since around 30 million infants are born via CS yearly, ${ }^{66}$ it is important that prospective studies, including a larger number of inclusions validate our observation that antenatally antibiotic exposure in CS born infants does not seem to impact early life microbiota development and does not have longterm health consequences.

\section{Author affiliations \\ 'Department of Paediatric Gastroenterology, Amsterdam UMC Locatie VUmc, Amsterdam, The Netherlands \\ ${ }^{2}$ Department of Paediatric Gastroenterology, Amsterdam UMC Locatie AMC, Amsterdam, The Netherlands \\ ${ }^{3}$ Nutricia Research Center, Utrecht, The Netherlands \\ ${ }^{4}$ Department of Paediatrics, Dalhousie University, Halifax, Nova Scotia, Canada ${ }^{5}$ Department of Neonatology, Amsterdam UMC Locatie AMC, Amsterdam, The Netherlands \\ ${ }^{6}$ Department of Obstetrics and Gynaecology, Reproduction and Development, Amsterdam UMC Locatie VUmc, Amsterdam, The Netherlands \\ Department of Gastroenterology and Hepatology, Amsterdam University Medical Centres, Amsterdam, The Netherlands \\ ${ }^{8}$ Department of Pharmaceutical Analysis, University of Groningen Groningen Research Institute of Pharmacy, Groningen, The Netherlands \\ ${ }^{9}$ Department of Clinical Pharmacy and Pharmacology, University Medical Centre Groningen, Groningen, The Netherlands \\ ${ }^{10}$ Laboratory of Microbiology, Wageningen University \& Research, Wageningen, The Netherlands}

Acknowledgements The authors would like to thank all patients and staff for their contributions to this trial. The authors would like to thank Heleen de Weerd for her guidance and help with the bio informatics involved in this study.

Contributors TdM and JdV designed the study and had responsibility overall of the study. DB, NS, LV and TD included participants and collected data and material. $J V L$ supervised the performance of whole metagenome sequencing. GR supervised the performance of $16 \mathrm{~S}$ rRNA gene sequencing analysis and the statistics of the sequenced data. DT performed the cefuroxime analysis. TD and TdM led the writing of this editorial, and all other authors contributed equally with comments and feedback. TdM is the guarantor for this paper. All authors read and approved the final manuscript. The corresponding author attests that all listed authors meet authorship criteria of the ICMJE Recommendations and that no others meeting the criteria have been omitted.

Funding This research was partially supported by Nutricia Research (Utrecht, the Netherlands) by financing costs for the microbiota analysis and by Stichting Zeldzame Ziektefonds. Whole metagenome sequencing was supported by a Canadian Institutes of Health Research (CIHR)- Canadian Association of Gastroenterology-Crohn's Colitis Canada New Investigator Award (2015-2019), a Canada Research Chair Tier 2 in Translational Microbiomics (2018-2019) and a Canadian Foundation of Innovation John R. Evans Leadership fund (awards \#35235 and \#36764) for JvL. Researchers from the funding sources declare their independence. All authors had full access to all of the data (including statistical reports and tables) in the study and take responsibility for the integrity of the data and the accuracy of the data analysis. The guidelines on good publication practice (GPP2) have been followed.

Disclaimer The funding sources had no role in the design and conduct of the study; collection, management, analysis, and interpretation of the data; preparation, review, or approval of the manuscript; and decision to submit the manuscript for publication.

Competing interests All authors have completed the International Committee of Medical Journal Editors (ICMJE) uniform disclosure form at http://www.icmje.org/ conflicts-of-interest/. Authors AE, ST, JK and GR are employees of Danone Nutricia Research which partially funded the Clinical Trial. TdM has served as a speaker for Danone Nutricia Research and Mead Johnson. NdB has served as a speaker for AbbVie and MSD. He has served as consultant and/or principal investigator for TEVA Pharma BV and Takeda. He has received a (unrestricted) research grant from Dr. Falk, TEVA Pharma BV and Takeda. The other authors have no financial disclosures that would be a potential conflict of interest.

\section{Patient consent for publication Not required.}

Provenance and peer review Not commissioned; externally peer reviewed.

Data availability statement Data are available upon reasonable request.

Supplemental material This content has been supplied by the author(s). It has not been vetted by BMJ Publishing Group Limited (BMJ) and may not have been peer-reviewed. Any opinions or recommendations discussed are solely those of the author(s) and are not endorsed by BMJ. BMJ disclaims all liability and responsibility arising from any reliance placed on the content. Where the content includes any translated material, BMJ does not warrant the accuracy and reliability of the translations (including but not limited to local regulations, clinical guidelines, terminology, drug names and drug dosages), and is not responsible for any error and/or omissions arising from translation and adaptation or otherwise.

Open access This is an open access article distributed in accordance with the Creative Commons Attribution Non Commercial (CC BY-NC 4.0) license, which permits others to distribute, remix, adapt, build upon this work non-commercially, and license their derivative works on different terms, provided the original work is properly cited, appropriate credit is given, any changes made indicated, and the use is non-commercial. See: http://creativecommons.org/licenses/by-nc/4.0/.

\section{ORCID iDs}

Thomas Dierikx http://orcid.org/0000-0003-0790-0987

Johan van Limbergen http://orcid.org/0000-0001-5822-4371

\section{REFERENCES}

1 Gilbert JA, Blaser MJ, Caporaso JG. Current understanding of the human microbiome. Nat Med 2018;24:392-400.

2 Bartlett JG, Chang TW, Gurwith M. Antibiotic-associated pseudomembranous colitis due to toxin-producing clostridia. N Engl J Med 1978;298:531-4.

3 Esaiassen E, Fjalstad JW, Juvet LK. Antibiotic exposure in neonates and early adverse outcomes: a systematic review and meta-analysis. J Antimicrob Chemother 2017;72:1858-70.

4 Vatanen T, Franzosa EA, Schwager R. The human gut microbiome in early-onset type 1 diabetes from the TEDDY study. Nature 2018;562:589-94.

5 Galazzo G, van Best N, Bervoets L. Development of the microbiota and associations with birth mode, diet, and atopic disorders in a longitudinal analysis of stool samples, collected from infancy through early childhood. Gastroenterology 2020;158:1584-96.

6 Lynch SV, Pedersen 0 . The human intestinal microbiome in health and disease. New England Journal of Medicine 2016:375:2369-79.

7 National Institute for Health and Clinical Excellence. Caesarean Section (NICE guideline 132). Updated september 2019, 2011. Available: https://www.nice.org.uk/ guidance/cg132 [Accessed Mar 2020].

8 National Institute for Health and Clinical Excellence. Neonatal infection (early onset): antibiotics for prevention and treatment (NICE guideline 149), 2012. Available: https://www.nice.org.uk/guidance/CG149 [Accessed Mar 2020]. 
9 Bedford Russell AR, Murch SH. Could peripartum antibiotics have delayed health consequences for the infant? BJOG : an international journal of obstetrics and gynaecology 2006;113:758-65.

10 Alonso-Ojembarrena A, Martínez-Díaz JV, Lechuga-Sancho AM. Broad spectrum antibiotics in newborns increase multi-drug resistant infections. Journal of chemotherapy 2019;31:81-5.

11 Saari A, Virta LJ, Sankilampi U. Antibiotic exposure in infancy and risk of being overweight in the first 24 months of life. Pediatrics 2015;135:617-26.

12 Mackeen AD, Packard RE, Ota E. Timing of intravenous prophylactic antibiotics for preventing postpartum infectious morbidity in women undergoing cesarean delivery. Cochrane Database Syst Rev 2014;12:CD009516.

13 Dierikx TH, Berkhout DJC, Visser L. The influence of timing of maternal administration of antibiotics during cesarean section on the intestinal microbial colonization in infants (MAMI-trial): study protocol for a randomised controlled trial. Trials 2019;20:479.

14 National Institute for Health and Clinical Excellence. Caesarean Section (NICE guideline 13), 2004. Available: https://www.nice.org.uk/guidance/CG13

15 Dierikx TH, Visser DH, Benninga MA. The influence of prenatal and intrapartum antibiotics on intestinal microbiota colonisation in infants: a systematic review. J Infect 2020 (published Online First: 2020/05/12).

16 Nogacka A, Salazar N, Suarez M. Impact of intrapartum antimicrobial prophylaxis upon the intestinal microbiota and the prevalence of antibiotic resistance genes in vaginally delivered full-term neonates. Microbiome 2017;5:93.

17 de Meij TG, Budding AE, de Groot EF. Composition and stability of intestinal microbiota of healthy children within a Dutch population. Faseb j 2016:30:1512-22

18 Klindworth A, Pruesse E, Schweer T. Evaluation of general 16S ribosomal RNA gene PCR primers for classical and next-generation sequencing-based diversity studies. Nucleic Acids Res 2013;41:e1.

19 Caporaso JG, Kuczynski J, Stombaugh J. QIIME allows analysis of high-throughput community sequencing data. Nat Methods 2010;7:335-6.

20 Schloss PD, Westcott SL, Ryabin T. Introducing mothur: open-source, platformindependent, community-supported software for describing and comparing microbial communities. Appl Environ Microbiol 2009;75:7537-41.

21 Rognes T, Flouri T, Nichols B. VSEARCH: a versatile open source tool for metagenomics. PeerJ 2016;4:e2584

22 Haas BJ, Gevers D, Earl AM. Chimeric 16S rRNA sequence formation and detection in Sanger and 454-pyrosequenced PCR amplicons. Genome Res 2011;21:494-504.

23 Amir A, McDonald D, Navas-Molina JA, et al. Deblur rapidly resolves single-nucleotide community sequence patterns. mSystems 2017;2.

24 Bolyen E, Rideout JR, Dillon M. QIIME 2: reproducible, interactive, scalable, and extensible microbiome data science 2018

25 Wang Q, Garrity GM, Tiedje JM. Naive Bayesian classifier for rapid assignment of rRNA sequences into the new bacterial taxonomy. App/ Environ Microbiol 2007;73:5261-7.

26 Pruesse E, Quast C, Knittel K. SILVA: a comprehensive online resource for quality checked and aligned ribosomal RNA sequence data compatible with ARB. Nucleic Acids Res 2007;35:7188-96.

27 McMurdie PJ, Holmes S. phyloseq: an R package for reproducible interactive analysis and graphics of microbiome census data. PLoS One 2013;8:e61217-e17.

28 Vegan: community ecology package, 2018. Available: https://CRAN.R-project.org/package= vegan

29 R: a language and environment for statistical computing, 2018. Available: https:// www.R-project.org/

30 Caporaso JG, Lauber CL, Walters WA. Ultra-high-throughput microbial community analysis on the Illumina HiSeq and MiSeq platforms. Isme j 2012;6:1621-4.

31 Gilbert JA, Meyer F, Jansson J. The Earth Microbiome Project: Meeting report of the " EMP meeting on sample selection and acquisition" at Argonne National Laboratory October 6 2010. Stand Genomic Sci 2010;3:249-53.

32 Liang G, Zhao C, Zhang H. The stepwise assembly of the neonatal virome is modulated by breastfeeding. Nature 2020;581:470-4.

33 Bittinger K, Zhao C, Li Y. Bacterial colonization reprograms the neonatal gut metabolome. Nature microbiology 2020;5:838-47.

34 Stewart CJ, Ajami NJ, O'Brien JL. Temporal development of the gut microbiome in early childhood from the TEDDY study. Nature 2018;562:583-8.

35 Keegan KP, Glass EM, Meyer F. MG-RAST, a Metagenomics service for analysis of microbial community structure and function. Methods in molecular biology 2016;1399:207-33.

36 Meyer F, Paarmann D, D'Souza M. The metagenomics RAST server - a public resource for the automatic phylogenetic and functional analysis of metagenomes. BMC Bioinformatics 2008;9:386.

37 DeSantis TZ, Hugenholtz P, Larsen N. Greengenes, a chimera-checked 16S rRNA gene database and workbench compatible with ARB. Appl Environ Microbiol 2006;72:5069-72
38 Cole JR, Chai B, Marsh TL. The ribosomal database project (RDP-II): previewing a new autoaligner that allows regular updates and the new prokaryotic taxonomy. Nucleic Acids Res 2003;31:442-3

39 Wilke A, Harrison T, Wilkening J. The M5nr: a novel non-redundant database containing protein sequences and annotations from multiple sources and associated tools. BMC Bioinformatics 2012;13:141

40 Arango-Argoty G, Garner E, Pruden A. DeepARG: a deep learning approach for predicting antibiotic resistance genes from metagenomic data. Microbiome 2018;6:23.

41 Alcock BP, Raphenya AR, TTY L. CARD 2020: antibiotic resistome surveillance with the comprehensive antibiotic resistance database. Nucleic Acids Res 2020;48:D517-25

42 Lin H, Peddada SD. Analysis of compositions of microbiomes with bias correction. Nat Commun 2020;11:3514

43 Benjamini Y, Hochberg Y. Controlling the false discovery rate: a practical and powerful approach to multiple testing. Journal of the Royal Statistical Society Series $B$ 1995:57:289-300

44 Braak ter CJF, Smilauer P. Canoco reference manual and user's guide: software for ordination version 5.0, (2012). Available: https://library.wur.n//WebQuery/wurpubs/431861

45 Overbeek R, Begley T, Butler RM. The subsystems approach to genome annotation and its use in the project to annotate 1000 genomes. Nucleic Acids Res 2005;33:5691-702.

46 The European Committee on Antimicrobial Susceptibility Testing (EUCAST). Breakpoin tables for interpretation of MICs and zone diameters [Version 5.26]. Available: https:// eucast.org/mic_distributions_and_ecoffs/ [Accessed Aug 2020].

47 Betran $A P, Y e$ J, Moller $A B$. The increasing trend in caesarean section rates: global, regional and national estimates: 1990-2014. PLoS One 2016;11:e0148343.

48 Shao Y, Forster SC, Tsaliki E. Stunted microbiota and opportunistic pathogen colonization in caesarean-section birth. Nature 2019;574:117-21.

49 Jakobsson HE, Abrahamsson TR, Jenmalm MC. Decreased gut microbiota diversity, delayed Bacteroidetes colonisation and reduced Th1 responses in infants delivered by caesarean section. Gut 2014;63:559-66.

50 Saturio S, Nogacka AM, Suárez M. Early-life development of the bifidobacterial community in the infant gut. Int J Mol Sci 2021;22:3382.

51 O'Callaghan A, van Sinderen D. Bifidobacteria and their role as members of the human gut microbiota. Front Microbiol 2016;7:925-25.

52 Fukuda S, Toh $\mathrm{H}$, Hase K. Bifidobacteria can protect from enteropathogenic infection through production of acetate. Nature 2011;469:543-7.

53 Rudin A, Lundell A-C. Infant B cell memory and gut bacterial colonization. Gut Microbes 2012;3:474-5

54 Tamana SK, Tun HM, Konya T. Bacteroides-dominant gut microbiome of late infancy is associated with enhanced neurodevelopment. Gut Microbes 2021;13:1-17.

55 Arrieta M-C, Stiemsma LT, Amenyogbe N. The intestinal microbiome in early life: health and disease. Front Immunol 2014:5:427-27.

56 Fjalstad JW, Esaiassen E, Juvet LK. Antibiotic therapy in neonates and impact on gut microbiota and antibiotic resistance development: a systematic review. J Antimicrob Chemother 2018;73:569-80.

57 Charteris WP, Kelly PM, Morelli L. Antibiotic susceptibility of potentially probiotic Bifidobacterium isolates from the human gastrointestinal tract. Lett Appl Microbiol 1998:26:333-7.

58 Neut C, Mahieux S, Dubreuil LJ. Antibiotic susceptibility of probiotic strains: is it reasonable to combine probiotics with antibiotics? Medecine et maladies infectieuses 2017:47:477-83.

59 Kamal SS, Hyldig N, Krych L. Impact of early exposure to cefuroxime on the composition of the gut microbiota in infants following cesarean delivery. $J$ Pediatr 2019;210:99-105.

60 Escobar-Zepeda A, Godoy-Lozano EE, Raggi L. Analysis of sequencing strategies and tools for taxonomic annotation: defining standards for progressive metagenomics. Sci Rep 2018;8:12034

61 Laudadio I, Fulci V, Palone F. Quantitative assessment of shotgun Metagenomics and $16 \mathrm{~S}$ rDNA amplicon sequencing in the study of human gut microbiome. Omics 2018;22:248-54.

62 Ranjan R, Rani A, Metwally A. Analysis of the microbiome: advantages of whole genome shotgun versus $16 \mathrm{~S}$ amplicon sequencing. Biochem Biophys Res Commun 2016:469:967-77.

63 Tessler M, Neumann JS, Afshinnekoo E. Large-scale differences in microbial biodiversity discovery between $16 \mathrm{~S}$ amplicon and shotgun sequencing. Sci Rep 2017;7:6589.

64 Visconti A, Le Roy Cl, Rosa F. Interplay between the human gut microbiome and host metabolism. Nat Commun 2019:10:4505.

65 Acinas SG, Sarma-Rupavtarm R, Klepac-Ceraj V. PCR-induced sequence artifacts and bias: insights from comparison of two 16S rRNA clone libraries constructed from the same sample. Appl Environ Microbiol 2005;71:8966-9.

66 Boerma T, Ronsmans C, Melesse DY. Global epidemiology of use of and disparities in caesarean sections, Lancet 2018:392:1341-8. 